\title{
Isolated intramedullary neurosarcoidosis of the thoracic spine: case report and review of the literature
}

\author{
Bradley S. Duhon $\cdot$ Lubdha Shah $\cdot$ Meic H. Schmidt
}

Received: 16 December 2010/Revised: 6 April 2011/Accepted: 6 May 2011/Published online: 20 May 2011

(c) Springer-Verlag 2011

\begin{abstract}
Sarcoidosis can involve the central nervous system in approximately $5-15 \%$ of cases. Any part of the nervous system can be involved, so presentation can be quite varied. Isolated disease of the spinal cord is even less common and reports are limited to single cases and small series. Although in the setting of systemic disease the diagnosis can be made with skin or lymph node biopsy, isolated disease of the spinal cord presents a diagnostic challenge. We present a case of isolated intramedullary neurosarcoidosis of the distal thoracic spinal cord presenting with posterior column dysfunction. Imaging demonstrated T2 changes in the patient's lower thoracic cord adjacent to disc herniation. Over time, however, his symptoms progressed despite decompression, and the abnormal region began to exhibit focal contrast enhancement. The persistence of symptoms as well as the new enhancement led us to perform a spinal cord biopsy, which demonstrated histopathological findings consistent with sarcoidosis. Further workup failed to reveal any evidence of systemic disease. Intramedullary sarcoidosis without systemic sarcoidosis is extremely rare. With its variable imaging appearance and inconsistent clinical manifestations, it can be difficult to diagnose. It should be considered
\end{abstract}

B. S. Duhon · M. H. Schmidt $(\square)$

Department of Neurosurgery, Clinical Neurosciences Center, University of Utah, 175 N. Medical Dr. East, Salt Lake City, UT 84132, USA

e-mail: neuropub@hsc.utah.edu

L. Shah

Department of Radiology, University of Utah,

Salt Lake City, UT, USA in the differential diagnosis of a mass-like intramedullary lesion with progressive symptoms. Biopsy with histopathological correlation may be the only definite management option.

Keywords Sarcoid · Neurosarcoidosis - Intramedullary · Spine - Isolated

\section{Introduction}

Sarcoidosis is a systemic, granulomatous disease most commonly involving the lymph nodes, skin, lungs, and eyes. Central nervous system (CNS) involvement (neurosarcoidosis) occurs in $5-15 \%$ of cases, and $6-8 \%$ of patients with neurosarcoidosis have spinal cord involvement; however, among all patients with sarcoidosis, the incidence of isolated spinal cord is only $0.43 \%[3,17]$.

The cause of the disease process is unknown, and the hallmark of sarcoidosis is the presence of noncaseating granulomas found throughout the body [15, 31]. Although radiographic findings and laboratory values may lead to the diagnosis, biopsy of the involved organ systems is the gold standard [7]. Spinal neurosarcoidosis can mimic an inflammatory demyelinating process both clinically and on imaging studies, particularly on magnetic resonance imaging (MRI) [18].

We present a case of isolated intramedullary sarcoidosis involving the distal thoracic spinal cord, which presented as lower extremity myelopathy and required spinal cord biopsy for diagnosis. Thorough workup had revealed no evidence of systemic disease, demonstrating an unusual presentation of sarcoidosis. We provide a review of imaging, laboratory studies, and histopathological findings useful for diagnosis of spinal neurosarcoidosis. 


\section{Case report}

This 48-year-old man had a history of lower back pain of 1.5-year duration. The pain was centered at the thoracolumbar region and was associated with shock-like sensations down the legs when the patient coughed or strained (in a classic "Lhermitte's" fashion). The pain progressed to include leg numbness (more pronounced on the right side than on the left), tingling, and weakness. He described a "buzzing" sensation with poor position-sense in his lower extremities. After 1 year of symptoms, the patient was evaluated by an outside physician, and nonenhanced MRI demonstrated a right paracentral disc protrusion at T10-11 resulting in moderate central canal stenosis and deformity of the ventral cord. In addition, the cord showed mild fusiform expansion and hyperintensity on T2-weighted MRI, which was concerning for spondylotic compressive myelopathy (Fig. 1).

The patient underwent thoracic laminectomy and fusion to decompress the stenotic level. Despite surgery, his symptoms progressed until he began to have difficulty with walking. At the time of symptom onset, he was able to run 1.5 miles, but by the time of presentation he was having significant difficulty with ambulation and now required a cane. The progression of symptoms prompted referral to our institution 5 months after surgery.

Upon examination, the patient demonstrated full strength on confrontational testing but had difficulty with tandem gait. In addition, he had positive findings on Romberg testing. He had normal reflexes in his left leg and right patella, but an absent right Achilles reflex. His toes were downgoing bilaterally, and he had no clonus. His sensory examination revealed decreased vibratory sense down to the knees bilaterally and decreased proprioception in his left lower extremity. These neurologic findings prompted follow-up MRI with contrast enhancement. Enhanced MRI exhibited persistent mild expansion of a short segment of the distal thoracic cord at the T10-T11 level with ill-defined intramedullary $\mathrm{T} 2$ hyperintensity and patchy enhancement (Fig. 2).

There was also persistent moderate canal stenosis at the T10-T11 level. Because the clinical symptoms and neuroimaging abnormalities had persisted despite surgical decompression, the diagnosis of spondylotic compressive myelopathy was questioned and other differential entities such as neoplasm, inflammatory disease, and demyelinating disease were considered.

Initial laboratory work-up including lumbar puncture revealed that the patient had a mildly elevated protein level (55 mg/dL), a normal IgG index $(0.5 \mathrm{mg} / \mathrm{dL})$, negative oligoclonal bands, normal angiotensin converting enzyme (ACE) level (2.0 U/L), and no malignant cells on CSF cytology studies. His CSF also demonstrated 29 red blood cells and 6 white blood cells per $\mu \mathrm{L}$ with $99 \%$ lymphocytes. Serum ACE was ultimately found to be elevated to $71 \mathrm{U} / \mathrm{L}$, but it was only drawn after the histopathological diagnosis had been made via biopsy.

Because it was not possible to make a definitive diagnosis based on laboratory studies, a repeat thoracic laminectomy for spinal cord biopsy was offered 9 months after the patient's previous surgery. Despite the risks of an intramedullary biopsy, this was thought to be the best option for diagnosis. Intraoperatively, the thoracic cord had a normal external appearance with the exception of its significantly enlarged size. A midline dorsal myelotomy was created, revealing a slightly gray appearance of the
Fig. 1 a Sagittal T2-weighted MRI demonstrates mild fusiform enlargement of the spinal cord extending from the T9-T10 level to the T11-T12 level. b Axial T2-weighted MRI reveals central cord $\mathrm{T} 2$ hyperintensity and mild cord expansion
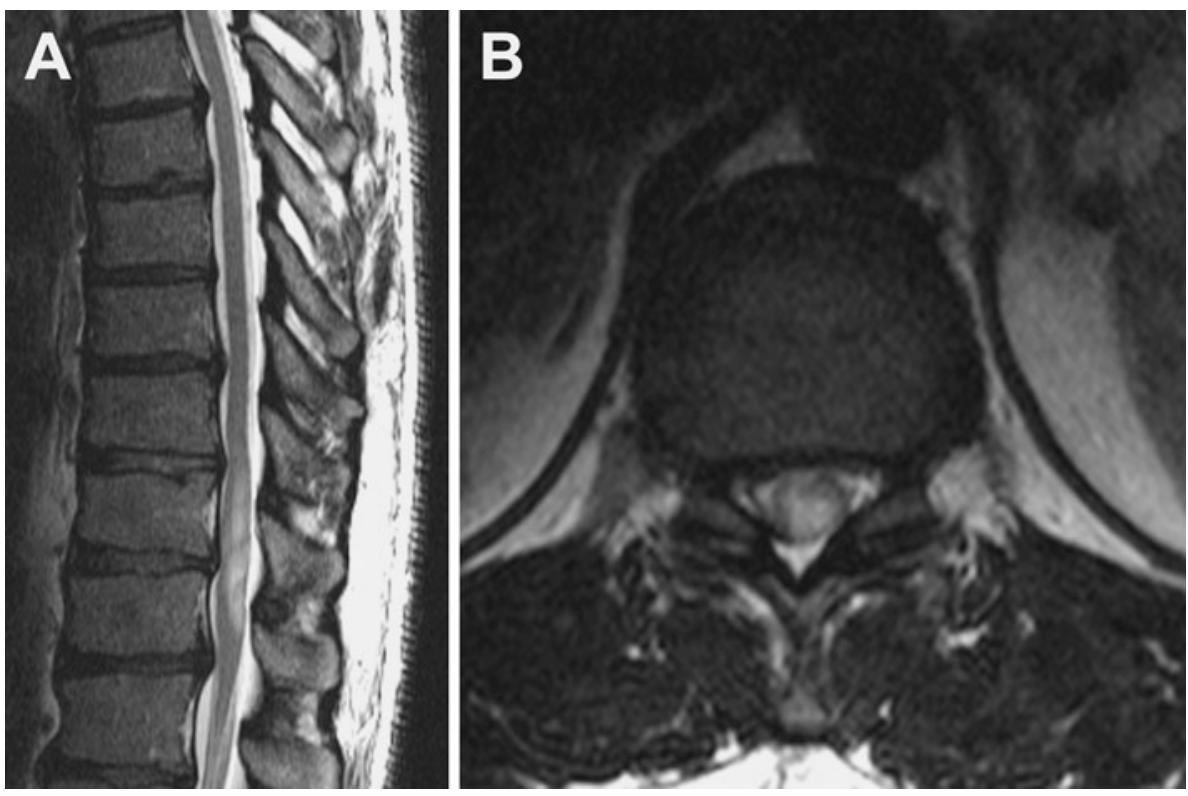
Fig. 2 a Sagittal T2-weighted MRI shows persistent mild cord expansion at the T10-T11 level. There is central T2 hyperintensity spanning the T9-T10 to the T10-T11 levels. The signal abnormality has tapered polar morphology. b Sagittal T1-weighted contrastenhanced MRI depicts patchy intramedullary enhancement at the T10-T11 level. No leptomeningeal enhancement is observed
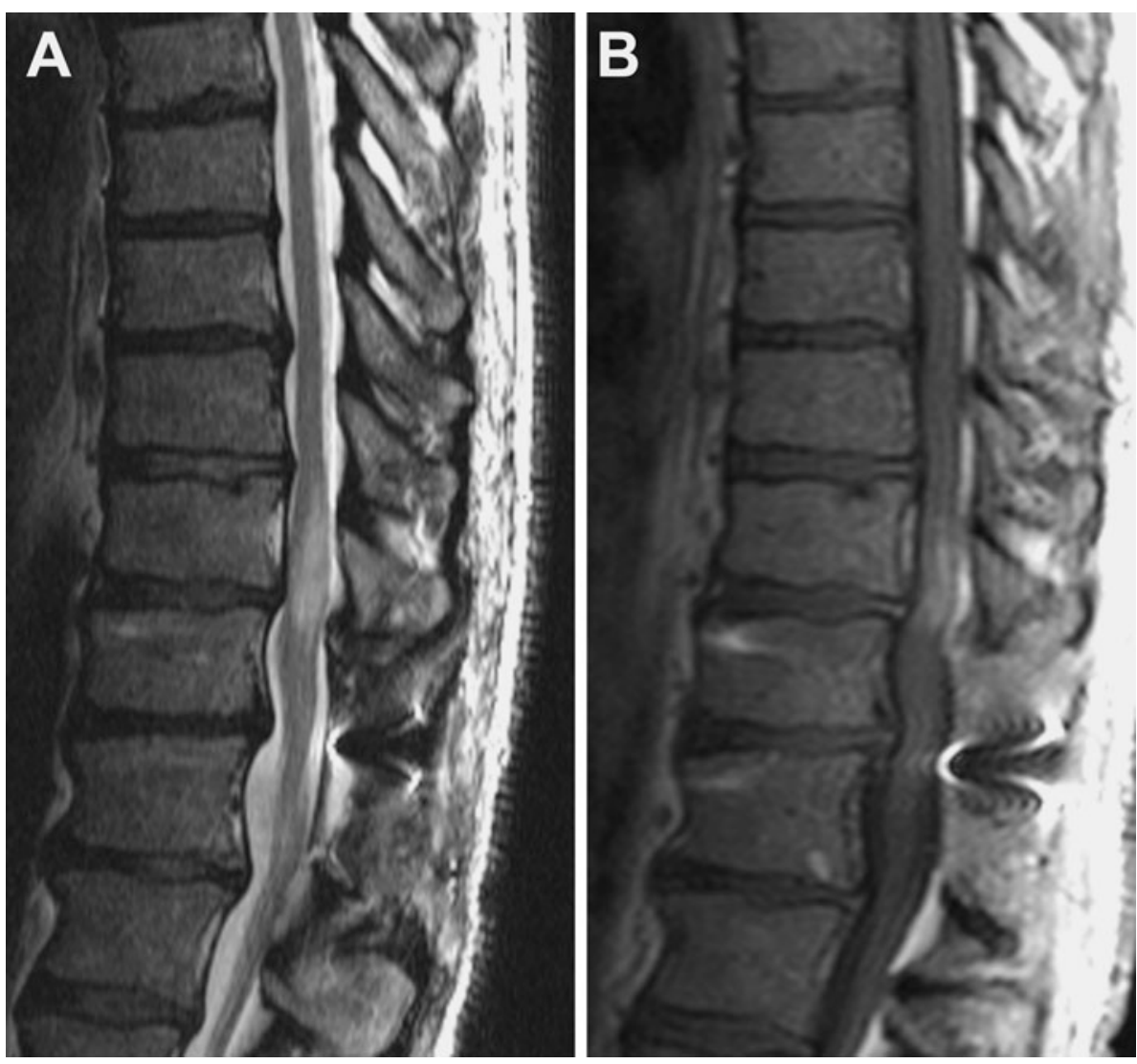

spinal cord internally. Specimens were sent for pathological analysis and, after dural closure, a posterior arthrodesis with pedicle screw fixation was performed from T11 to L1 (Fig. 3).

The patient initially had a worsening of his sensation and proprioception in his lower extremities, but this improved throughout his postoperative stay, and he was walking independently upon discharge 4 days later. At his 1-month follow-up visit, he exhibited full strength in his legs, and his sensory deficits had returned to his preoperative state but no better. Results from the biopsy demonstrated nonnecrotizing granulomatous disease consistent with sarcoidosis (Fig. 4).

After the histological diagnosis, treatment was initiated with prednisone and azathioprine [1]. The patient's symptoms improved over the next 3 months. At 1-year follow-up, the thoracic MRI scan showed near-resolution of the T2 hyperintensity and cord expansion in the distal spinal cord. In addition, the patient's symptoms were stabilized with mild numbness in his feet. At the most recent follow-up at 18 months, he had discontinued use of all immunosuppressants against medical advice because of the side effects. He continued to have significant "buzzing" in his legs with exertion, but this was relatively well controlled with gabapentin. $\mathrm{He}$ is scheduled to undergo imaging yearly.

\section{Discussion}

Neurosarcoidosis was first reported in 1904 [32]. Since then, it has become a well-described manifestation of sarcoidosis. Spinal cord involvement is more prevalent in the cervical spine (56\%) than in the thoracic $(37 \%)$ and lumbosacral (7\%) spine [16, 17]. Spinal sarcoidosis can present with varied symptoms, including weakness, paresthesias, myelopathy, demyelinating syndrome [18], facial nerve paralysis [28], bowel, bladder, or sexual disturbances, back pain $[10,26]$, or even radicular pain [30]. The characteristic demographic of spinal sarcoidosis is a male patient, 20-40 years of age, of Northern European or African descent. In systemic sarcoidosis there is a slight female predominance.

The imaging findings of CNS sarcoidosis can mimic other disease processes such as multiple sclerosis, metastasis, primary glial neoplasms, and extra-axial neoplasms. The imaging spectrum for neurosarcoidosis in the brain includes periventricular and subcortical white matter lesions on fluid-attenuated inversion recovery (FLAIR) and T2-weighted images, multiple supra- and infratentorial enhancing lesions, solitary intra- or extra-axial mass, and leptomeningeal enhancement [28, 33]. Spinal sarcoidosis most commonly demonstrates smooth or nodular leptomeningeal enhancement with patchy peripheral cord 
Fig. 3 Anteroposterior (a) and lateral (b) radiographs demonstrate posterior decompression with laminectomies at the T11, T12, and L1 levels. Pedicle screw fixation is visible at these levels
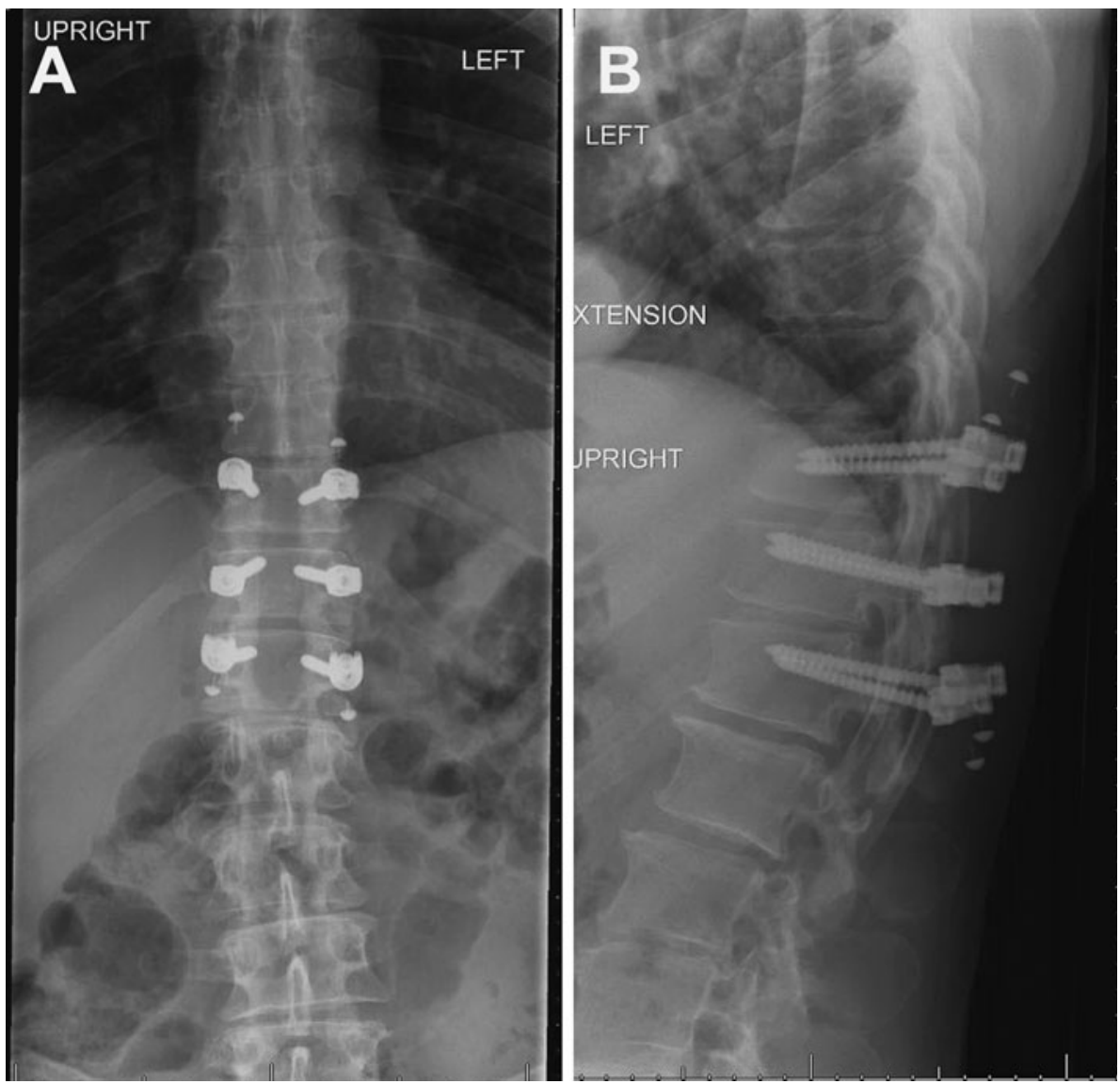

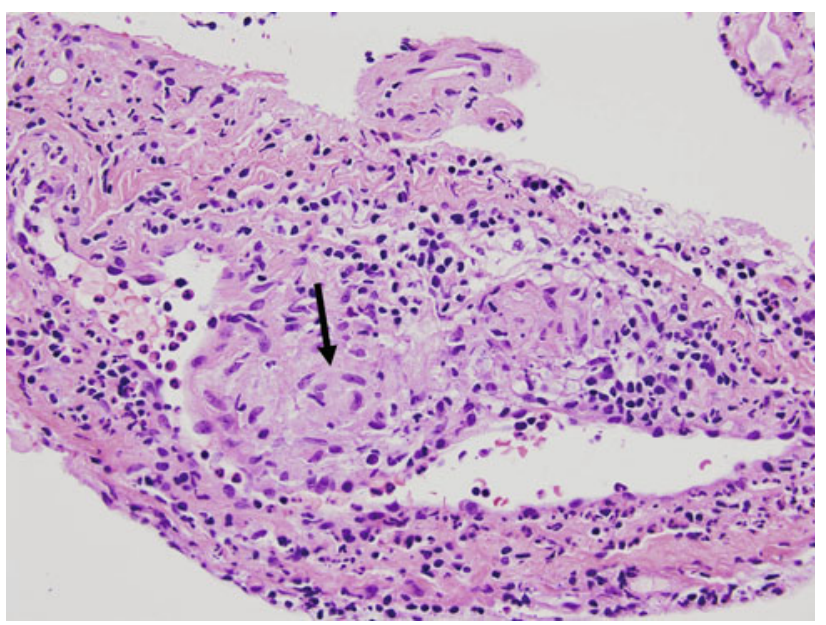

Fig. 4 High-magnification hematoxylin and eosin-stained section demonstrating perivascular noncaseating granulomas (arrow). There is also a lymphocytic infiltration of the surrounding tissue

enhancement because of infiltration of the perivascular spaces; however, spinal sarcoidosis has a myriad of imaging manifestations from diffuse intramedullary $\mathrm{T} 2$ hyperintensity to mass-like intramedullary enhancement. There may be osseous involvement with multiple lytic or mixed lytic-sclerotic lesions. Fusiform cord enlargement initially is followed by atrophy in the chronic stages. The combination of leptomeningeal and peripheral intramedullary enhancement is most suggestive of spinal sarcoidosis. In isolated spinal cord involvement, meningeal or parenchymal biopsy may be required. Treatment with steroids may result in decreased enhancement. Although many sarcoid-related MRI abnormalities are not associated with correlating symptoms, there is a high degree of concordance between changes in clinical symptoms and MRI abnormalities, particularly in the cranial vault and spinal cord [29].

The imaging differential diagnosis of isolated intramedullary sarcoidosis includes neoplasm, demyelinating processes (such as multiple sclerosis), post-infectious myelitis, spondylotic compressive myelopathy, lymphoma, cord ischemia, and paraneoplastic processes. When laboratory and radiographic findings are clear, the diagnosis may be suggested; however, histopathological confirmation is necessary to make a definitive diagnosis [33].

Laboratory work-up for spinal sarcoidosis should include CSF evaluation. CSF abnormalities are seen in approximately $80 \%$ of cases of neurosarcoidosis; however, these findings are quite varied and nonspecific [33]. Cell count, protein, glucose, and ACE levels should be 
obtained. Cell count typically shows few, if any, cells with a lymphocytic predominance. An elevated protein level is the most common finding. Even CSF oligoclonal bands can be identified in $36 \%$ of cases [24, 25, 33]. Serum and CSF ACE levels are often considered valuable in the diagnosis of neurosarcoidosis. Elevated levels of either are supportive of the diagnosis, but true-positive yield is quite low $(24-75 \%)[9,22,23]$. False positives can be seen in other disease processes including tuberculosis or malignancy $[2,21]$.

The histopathological hallmark of sarcoidosis is the noncaseating granuloma with multinucleated giant cells. CNS lesions may be smaller and have fewer giant cells within more poorly formed granulomas, which may increase the likelihood of a negative biopsy result $[18,27]$. Another pitfall of biopsy can be frozen sections mimicking tumor $[19,20]$. In cases in which leptomeningeal biopsies are obtained, the arachnoid is often infiltrated with lymphocytes and the typical granulomas. The proposed mechanism by which sarcoidosis becomes intramedullary is via extension through perivascular spaces [10].

Treatment of neurosarcoidosis is based on immunosuppressant therapy, with a goal of suppression rather than cure. Corticosteroids, such as prednisone, are the mainstay of immunosuppressant therapy, although stronger immunosuppressants, such as methotrexate, cyclosporine, cyclophosphamide, azathioprine, and chlorambucil, have also been used [1]. Most outcomes data in neurosarcoidosis are based on anecdotal reports and small series in the absence of prospective trials, with response to treatment being variable. Because of the suggested link between tumor necrosis factor- $\alpha$ (TNF- $\alpha)$ and disease, monoclonal antibodies to TNF- $\alpha$ have been studied for the treatment of refractory sarcoidosis $[5,6,8,11,12]$, although this therapy remains experimental. Surgery (especially for intramedullary lesions) is reserved for diagnostic biopsy, as surgical resection is morbid and not beneficial [4]. Localized radiation has also been used and may provide temporary relief [14]. Regardless of treatment, prognosis of neurosarcoidosis is quite variable. The presence of neurological symptoms at presentation has been associated with poor outcome, but steroid therapy in spinal disease may be linked to a more favorable outcome [13, 18].

Intramedullary sarcoidosis is a rare entity, and it is exceedingly rare without systemic sarcoidosis. Its variable imaging appearance and inconsistent clinical manifestations can pose a diagnostic dilemma. Although rare, isolated spinal cord neurosarcoidosis should be in the differential diagnosis when evaluating patients with myelopathy, especially if the patient does not improve or worsens despite an adequate decompression. In these patients, open biopsy may be indicated if there are no other sites of sarcoidosis on the work up.
Acknowledgments We thank Kristin Kraus, M.Sc., for editorial assistance and Steven Chin, M.D., for preparation of the pathology slides and photographs.

Conflict of interest None of the authors has any potential conflict of interest.

\section{References}

1. Agbogu BN, Stern BJ, Sewell C, Yang G (1995) Therapeutic considerations in patients with refractory neurosarcoidosis. Arch Neurol 52:875-879

2. Allen R, Mendelsohn FA, Csicsmann J, Weller RF, Hurley TH, Doyle AE (1980) A clinical evaluation of serum angiotensin converting enzyme in sarcoidosis. Aust N Z J Med 10:496-501

3. Bogousslavsky J, Hungerbuhler JP, Regli F, Graf HJ (1982) Subacute myelopathy as the presenting manifestation of sarcoidosis. Acta Neurochir (Wien) 65:193-197

4. Bose B (2002) Extramedullary sarcoid lesion mimicking intraspinal tumor. Spine J 2:381-385

5. Callejas-Rubio JL, Lopez-Perez L, Ortego-Centeno N (2008) Tumor necrosis factor-alpha inhibitor treatment for sarcoidosis. Ther Clin Risk Manag 4:1305-1313

6. Callejas-Rubio JL, Ortego-Centeno N, Lopez-Perez L, Benticuaga MN (2006) Treatment of therapy-resistant sarcoidosis with adalimumab. Clin Rheumatol 25:596-597. doi:10.1007/s10067005-0037-9

7. Caneparo D, Lucetti C, Nuti A, Cipriani G, Tessa C, Fazzi P, Bonuccelli U (2007) A case of sarcoidosis presenting as a non-specific intramedullary lesion. Eur J Neurol 14:346-349. doi:10.1111/j.1468-1331.2006.01527.x

8. Dai H, Guzman J, Chen B, Costabel U (2005) Production of soluble tumor necrosis factor receptors and tumor necrosis factoralpha by alveolar macrophages in sarcoidosis and extrinsic allergic alveolitis. Chest 127:251-256. doi:10.1378/chest.127.1.251

9. Dale JC, O'Brien JF (1999) Determination of angiotensin-converting enzyme levels in cerebrospinal fluid is not a useful test for the diagnosis of neurosarcoidosis. Mayo Clin Proc 74:535

10. Day AL, Sypert GW (1977) Spinal cord sarcoidosis. Ann Neurol 1:79-85. doi:10.1002/ana.410010108

11. Denys BG, Bogaerts Y, Coenegrachts KL, De Vriese AS (2007) Steroid-resistant sarcoidosis: is antagonism of TNF-alpha the answer? Clin Sci (Lond) 112:281-289. doi:10.1042/CS20060094

12. Dhaille F, Viseux V, Caudron A, Dadban A, Tribout C, Boumier P, Clabaut A, Lok C (2010) Cutaneous sarcoidosis occurring during anti-TNF-alpha treatment: report of two cases. Dermatology 220:234-237. doi:10.1159/000275676

13. Ferriby D, de Seze J, Stojkovic T, Hachulla E, Wallaert B, Destee A, Hatron PY, Vermersch P (2001) Long-term follow-up of neurosarcoidosis. Neurology 57:927-929

14. Gelwan MJ, Kellen RI, Burde RM, Kupersmith MJ (1988) Sarcoidosis of the anterior visual pathway: successes and failures. J Neurol Neurosurg Psychiatry 51:1473-1480

15. Hayat GR, Walton TP, Smith KR Jr, Martin DS, Manepalli AN (2001) Solitary intramedullary neurosarcoidosis: role of MRI in early detection. J Neuroimaging 11:66-70

16. Jaster JH, Dohan FC Jr, Bertorini TE, Bass JE, Monkemuller KE, Handorf CR, Gerald BE (1997) Solitary spinal cord sarcoidosis without other manifestations of systemic sarcoidosis. Clin Imaging 21:17-22

17. Junger SS, Stern BJ, Levine SR, Sipos E, Marti-Masso JF (1993) Intramedullary spinal sarcoidosis: clinical and magnetic resonance imaging characteristics. Neurology 43:333-337 
18. Kumar N, Frohman EM (2004) Spinal neurosarcoidosis mimicking an idiopathic inflammatory demyelinating syndrome. Arch Neurol 61:586-589. doi:10.1001/archneur.61.4.586

19. Levivier M, Baleriaux D, Matos C, Pirotte B, Brotchi J (1991) Sarcoid myelopathy. Neurology 41:1529-1530

20. Levivier M, Brotchi J, Baleriaux D, Pirotte B, Flament-Durand J (1991) Sarcoidosis presenting as an isolated intramedullary tumor. Neurosurgery 29:271-276

21. Lidar M, Dori A, Levy Y, Lidar Z, Chapman J, Langevitz P (2010) Sarcoidosis presenting as "corset-like" myelopathy: a description of six cases and literature review. Clin Rev Allergy Immunol 38:270-275. doi:10.1007/s12016-009-8156-8

22. Lieberman $\mathbf{J}$ (1975) Elevation of serum angiotensin-convertingenzyme (ACE) level in sarcoidosis. Am J Med 59:365-372

23. Lieberman J (1989) Enzymes in sarcoidosis. Angiotensin-converting-enzyme (ACE). Clin Lab Med 9:745-755

24. McLean BN, Miller D, Thompson EJ (1995) Oligoclonal banding of IgG in CSF, blood-brain barrier function, and MRI findings in patients with sarcoidosis, systemic lupus erythematosus, and Behcet's disease involving the nervous system. J Neurol Neurosurg Psychiatry 58:548-554

25. McLean BN, Mitchell DN, Thompson EJ (1990) Local synthesis of specific IgG in the cerebrospinal fluid of patients with neurosarcoidosis detected by antigen immunoblotting using Kveim material. J Neurol Sci 99:165-175
26. Nagai H, Ohtsubo K, Shimada H (1985) Sarcoidosis of the spinal cord. Report of an autopsy case and review of the literature. Acta Pathol Jpn 35:1007-1022

27. Nathan MP, Chase PH, Elguezabel A, Weinstein M (1976) Spinal cord sarcoidosis. N Y State J Med 76:748-752

28. Pickuth D, Heywang-Kobrunner SH (2000) Neurosarcoidosis: evaluation with MRI. J Neuroradiol 27:185-188

29. Shah R, Roberson GH, Cure JK (2009) Correlation of MR imaging findings and clinical manifestations in neurosarcoidosis. Am J Neuroradiol 30:953-961. doi:10.3174/ajnr.A1470

30. Shono T, Tamai M, Kobayashi M, Wakasaki H, Furuta H, Nakao T, Hanabusa T, Nishi M, Sasaki H, Nanjo K (2004) Neurosarcoidosis with spinal root pain as the first symptom. Intern Med 43:873-877

31. Thomas KW, Hunninghake GW (2003) Sarcoidosis. JAMA 289:3300-3303. doi:10.1001/jama.289.24.3300

32. Winkler M (1905) Beitrag zur Frage der Sarkoidose (Boeck). Arch Dermatol Syphilis 77:3-24

33. Zajicek JP, Scolding NJ, Foster O, Rovaris M, Evanson J, Moseley IF, Scadding JW, Thompson EJ, Chamoun V, Miller DH, McDonald WI, Mitchell D (1999) Central nervous system sarcoidosis-diagnosis and management. QJM 92:103-117 